\title{
ON HERSTEIN'S THEOREM CONCERNING THREE FIELDS
}

\author{
CARL FAITH ${ }^{1)}$
}

Let $L>K \geqq \emptyset, L \neq K$, be three fields such that: (1) $L / K$ is not purely inseparable, and (2) $L / \Phi$ is transcendental. Then Herstein's theorem [2] asserts the existence of $u \in L$ such that $f(u) \notin K$ for every non-constant polynomial $f(X) \in \mathscr{D}[X]$. Thus Herstein's theorem can be given the following equivalent form:

THEOREM (Herstein). If $L, K$, and $\emptyset$ are three fields satisfying (1) and (2), $L \neq K$, then there exists $u \in L$ which is transcendental over $\Phi$ such that $K \cap \emptyset[u]$ $=\emptyset$, where $\Phi[u]$ is the subring generated by $\emptyset$ and $u$.

The main part of Herstein's proof depends on a lemma of Nagata, Nakayama, and Tsuzuku in valuation theory of fields [On an existence lemma in valuation theory, Nagoya Math. Journal, vol. 6 (1953)]; the proof of this lemma in turn requires a knowledge of arithmetic in "algebraic number and function fields". In the present note I present an elementary proof of Herstein's theorem in which only the most basic properties of simple transcendental fields are used. In this development the result for the case $L=\emptyset(x)$ is sharpened: then there exists a polynomial $q=q(x) \in \emptyset[x]$ not in $\emptyset$ such that $K \cap \emptyset[q]=\emptyset$.

Herstein's elementary reduction to the pure transcendental case constitutes a reduction for the theorem as stated above so we can assume that $L=\emptyset(x)$. In this case it is known ${ }^{2)}$ that $K \cap \emptyset[x]$ is finitely generated over $\emptyset$ as a ring, for any intermediate field $K$. The proposition below gives a new proof and at the same time sharpens this result: Then $K \cap \emptyset[x]$ has a single generator over $\emptyset$.

Received March 22, 1961.

1) North Atlantic Treaty Organization (U.S.A.) Postdoctoral Fellow in the Mathematical Institute, Heidelberg University, on leave from Pennsylvania State University.

2) This is the one dimensional solution to Hilbert's Fourteenth Problem. See [4] for Zariski's generalization and solution to the one and two dimensional cases of Hilbert's problem. 
Proposition 1. Let $L=\Phi(x)$ be a simple transcendental field extension, and let $K=\Phi(H / G)$ be any intermediate field $d^{i)} \neq \Phi$, where $H, G \in \Phi[x],(H, G)=1$, and $H \notin \Phi$. Then a necessary and sufficient condition that $K \cap \Phi[x] \neq \emptyset$ is that $K=\emptyset(H)$. Then : $K \cap \emptyset[x]=\emptyset[H]$, and $G=a H+b$, with $a, b \in \emptyset$.

Proof. Let $P(x) \in \mathscr{D}[x], P(x) \notin \emptyset$, and assume that

$$
P=h(H / G) / g(H / G) \in K,
$$

where $h(X), g(X) \in \mathscr{D}[X] .(h, g)=1$, and $X$ a new indeterminant. It can, and will, be assumed that both $h(X)$ and $g(X)$ have leading coefficient $=1$. First suppose that $g(X) \in \emptyset$ (then $g(X)=1$ ) and write

$$
h(X)=X^{q} \sum_{i=0}^{k} a_{i} X^{i}
$$

where $a_{0} a_{k} \neq 0$. Then,

$$
G^{k+q} P=H^{q} \sum_{i=0}^{k} a_{i} G^{k-i} H^{i}
$$

Since $(H, G)=1$, necessarily $\left(G, \sum_{0}^{k} a_{i} G^{k-i} H^{i}\right)=1$. Since $H(x) \notin \mathscr{D}, k+q$ $=\operatorname{deg} H(x) \neq 0$. It follows, since $G$ divides the left side but is prime to the right side of (2), that $G \in \emptyset$, that is, $G=0 \cdot H+b \in \emptyset, K=\emptyset(H)$ as required.

Now assume that $g(X) \notin \emptyset$. I am indebted to R. Kiehl for the following neat proof of this case. Let $A$ denote the algebraic closure of $\emptyset$, and, over $A$, factor

$$
g(X)=\prod_{j=1}^{m}\left(X-b_{j}\right)
$$

Furthermore. over $A$,

$$
h(X)=\prod_{i=1}^{n}\left(X-a_{i}\right) \quad(\text { or, } h(X)=1 .)
$$

Hence, by (1) and (3),

$$
\left.\prod_{\jmath-1}^{m}\left(H-b_{j} G\right)=G^{t} \prod_{i=1}^{n}\left(H-a_{i} G\right) \quad \text { (or, }=G^{t}\right),
$$

where $t=m-n$ (or, $t=m$ ). Since $(H, G)=1$, clearly

$$
\left(H-b_{1} G, G\right)=1 \text {, }
$$

3) This is Lüroth's theorem [3, p. 126]. 
and,

$$
d_{i}=\left(H-b_{1} G, H-a_{j} G\right)=1 .
$$

To justify $(7)$, note, $($ since $(h, g)=1)$ that $a_{j} \neq b_{1}, j=1,2, \ldots m$, and write

$$
H-b_{1} G=\left(H-a_{j} G\right)+\left(a_{j}-b_{1}\right) G .
$$

From (8) it follows that $d_{j}$ divides $G$, whence $d_{j}$ divides $1=(H, G)$, that is, $d_{j}=1$. Now (5) - (7) show that $H-b_{1} G$ divides the left side but is prime (even in the case $h(X)=1$ ) to the right side of (5). Thus,

$$
H-b_{1} G=c_{1} \in A \text {. }
$$

Inspection of the coefficients in (9) reveals that $b_{1}, c_{1}, \in \Phi$, and, hence,

$$
G=b_{1}^{-1} H-c_{1} b_{1}^{-1}
$$

has the required form; $K=\emptyset(H)$.

Finally, since $K=\emptyset(H)$, (1) can be rewritten

$$
P(x)=h(H) / g(H) .
$$

The results above, and the form of (10), show, by assuming $G$ in (1) is a con-

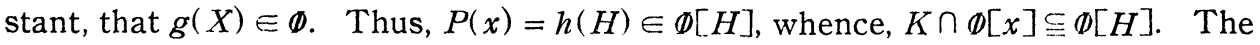
reverse inclusion is trivial, so that the last statement in the proposition is proved.

Lemma 2. Let $L=\emptyset(x)$ be a simple transcendental field extension, and let $K=\mathscr{D}(H)$, where $H=H(x) \in \mathscr{D}[x]$ is such that $x$ divides $H(x)$, and $K \cap \emptyset[x H]$ $\neq \emptyset$. Then, $H(x)=a x^{n}, a \in \emptyset$.

Proof. By Proposition 1, $\emptyset[H] \geqq \emptyset[x H] \cap K . \quad$ Let $f(X)=\sum_{0}^{m} a_{i} X^{i}, g(X)$ $=\sum_{0}^{m} b_{i} X^{i}$, where $m$ is chosen such that one of $a_{m}, b_{m} \neq 0$, be such that $f(x H)$ $=g(H) \in \Phi[x H]$ not in $\Phi$. Then

$$
0=g(H)-f(x H)=\sum_{0}^{n}\left(a_{i}-b_{i} x^{2}\right) H^{i} .
$$

If $q$ is the smallest integer such that one of $a_{q}, b_{q} \neq 0$, then (1) is divisible by $H^{q}$, so that

$$
0=\sum_{q}^{m}\left(a_{i}-b_{i} x^{i}\right) H^{i-q}
$$


From (2) one sees that $H$ divides $\left(a_{q}-b_{q} x^{q}\right)$. Since $H \notin \emptyset, q \neq 0$. Since $x$ divides $H$, necessarily $a_{q}=0$, whence $H$ divides $x^{q}$, that is, $H(x)=a x^{n}, a \in \Phi$, $n \leqq q$, as needed.

I am now in a position to complete the proof of Herstein's theorem (in its sharpened form in the pure transcendental case.)

THEOREM 3. If $L=\emptyset(x)$ is a simple transcendental field extension and if $K$ is any intermediate field $\neq L$ such that $L / K$ is not purely inseparable, then there exists $u \in \emptyset[x]$ not in $\emptyset$ such that $K \cap \emptyset[u]=\emptyset$.

Proof. If the theorem is denied, then by the proposition, $K=\emptyset(H)$ with $H=H(x) \in \mathscr{\Phi}[x]$. It can be assumed that $x$ divides $H(x)$. Now $K \cap \emptyset[x H] \neq \emptyset$, so that $K=\emptyset\left(x^{n}\right)$ by the lemma. Let $y=x-1$, note that $L=\emptyset(y)$, that $K=\emptyset\left(x^{n}-1\right)$, and assume that

$$
K \cap \emptyset\left[(x-1)\left(x^{n}-1\right)\right] \neq \emptyset,
$$

that is, that

$$
\Phi\left((y+1)^{n}-1\right) \cap \emptyset\left[y\left((y+1)^{n}-1\right)\right] \neq \emptyset .
$$

Then, since $y$ divides $(y+1)^{n}-1$, one can apply Lemma 2 again to see that

$$
(y+1)^{n}-1=y^{n},
$$

or,

$$
(y+1)^{n}=y^{n}+1 \text {, }
$$

which, since $n>1$, is possible only if $\Phi$ has characteristic $p$, and $n=p^{e}$. Then $K=\emptyset\left(x^{n}\right)=\emptyset\left(x^{p^{e}}\right)$, so that $L / K$ is purely inseparable, contrary to the hypothesis. This completes the proof.

Proposition 4. Let $L=\emptyset(x)$ be a simple transcendental field extension, and let $P, Q \in \Phi[x]$ be such that $\Phi(P) \cap \emptyset(Q) \neq \emptyset$. Then $\emptyset[P] \cap \emptyset[Q] \neq \emptyset$.

Proof. Let (1) $h(P) / g(P)=p(Q) / q(Q)$ be a nonconstant element in $M=\Phi(P) \cap \emptyset(Q)$, where $h(X), g(X), p(X), q(X) \in \emptyset[X]$, and $(h, g)=(p, q)=1$. Then, (2) $h(P) q(Q)=p(Q) g(P)$. Since $(q(Q), p(Q))=(h(P), g(P))=1$, it follows that (3) $h(P)=p(Q) \in M$, and (4) $q(Q)=g(P) \in M$, so that, by (1), one of (3) and (4) lies outside of $\emptyset$.

THEOREM 5. Let $L=\Phi(x)$ be a simple transcendeutal field extension, and let $K$ be an intermediate field such that $L \neq K$, and $L / K$ is not purely inseparable. 
Then, if $K=\emptyset(F(x))$, where $P(x) \in \Phi[x]$, there exists $Q(x) \in \Phi[x], Q(x) \notin \emptyset$, such that $K \cap \emptyset(Q)=K \cap \emptyset[Q]=\emptyset$.

Proof. If $\Phi(Q) \cap K \neq \emptyset$, for all $Q$ in $\mathscr{D}[x]$ not in $\Phi$, then by the proposition, $\mathscr{D}[Q] \cap K \neq \emptyset$, for all such $Q$. But this violates Theorem 3 , unless $L / K$ is purely inseparable. But this is ruled out by hypothesis, completing the proof.

In [1] Herstein's method of [2] is employed to show that the element $u$ in the statement of his theorem can be chosen such that

$$
K \cap \emptyset(u)=K \cap \emptyset[u]=\emptyset .
$$

Theorem 5, then, represents a special case of this more general result. It would be interesting therefore to know if the more general statement also has an elementary proof.

In [1] Herstein's theorem is used in the proof of the following result: If $A$ is a transcendental division algebra over the field $\emptyset$, and if $B$ is a subalgebra $\neq A$ such that to each $a \in A$ there corresponds a non-constant polynomial $f_{a}(x) \in \Phi[x]$ such that $f_{a}(a) \in B$, then $A$ is a field. A consequence of the present note is that this result now also has an elementary proof.

\section{REFERENCES}

[1] Carl Faith, A structure theory for semialgebraic extensions of division algebras, Journal für die reine und angewandte Mathematik, (1961).

[2] I. N. Herstein, A theorem concerning three fields, Canadian Journal of Mathematics, vol. 7 (1955), 202-203.

[3] B. L. van der Waerden, Algebra I, Vierte Auflage, Berlin-Göttingen-Heidelberg, 1955.

[4] O. Zariski, Interprétation algébrico-géométriques du quatorzième problème de Hilbert, Bull. Sci. Math. vol. 78 (1954), 155-168.

Institute for Advanced Study,

Princeton, N. J. 